\title{
Correction to: Induced cultivation pattern enhanced the phycoerythrin production in red alga Porphyridium purpureum
}

\author{
Yuanchao $\mathrm{Xu}^{1} \cdot$ Kailin Jiao ${ }^{2} \cdot$ Huichang Zhong ${ }^{3}$. Shengshan $\mathrm{Wu}^{2}$. Shih-Hsin $\mathrm{Ho}^{4} \cdot$ Xianhai Zeng $^{2,5}$. Jinglong $\mathrm{Li}^{1}$. \\ Xing Tang ${ }^{2,4} \cdot$ Yong Sun ${ }^{2,4} \cdot$ Lu Lin $^{2,4}$
}

Published online: 5 January 2020

○) Springer-Verlag GmbH Germany, part of Springer Nature 2020

\section{Correction to: Bioprocess and Biosystems Engineering https://doi.org/10.1007/s00449-019-02230-6}

The original version of the article unfortunately contained an error in Microalgae strain and culture medium section. Below is the corrected version.

\section{Microalgae strain and culture medium}

Porphyridium purpureum CoE2 The algal species used were purchased from the Freshwater Algae Culture Collection at the Institute of Hydrobiology of the Chinese Academy of Sciences. The strain was further induced, screened, and stored in artificial seawater (ASW) with periodical refreshment by the author's research group [20].

The original article can be found online at https://doi.org/10.1007/ s00449-019-02230-6.

Xianhai Zeng

xianhai.zeng@xmu.edu.cn

1 College of Biological Engineering, Qilu University of Technology (Shandong Academy of Sciences), Jinan 250353, China

2 College of Energy, Xiamen University, Xiamen 361102, China

3 Xiamen Huison Biotech Co., Ltd., No. 1337, Tongji Rd, Tongji Industrial District, Xiamen 361100, China

4 State Key Laboratory of Urban Water Resource and Environment, School of Municipal and Environmental Engineering, Harbin Institute of Technology, Harbin 150001, People's Republic of China

5 Fujian Engineering and Research Center of Clean and High-Valued Technologies for Biomass, Xiamen Key Laboratory of Clean and High-valued Utilization for Biomass, Xiamen University, Xiamen 361102, China
Publisher's Note Springer Nature remains neutral with regard to jurisdictional claims in published maps and institutional affiliations. 